\title{
EFEITO DO EXERCÍCIO DE FORÇA NA COMPOSIÇÃO CORPORAL DE RATOS OBESOS
}

João Henrique Lyrio Machado ${ }^{1}$, Rafael Junges Moreira ${ }^{1}$, Alan José Barbosa Magalhães ${ }^{1}$, Robson Chacon Castoldi ${ }^{1,2,3}$, Guilherme Akio Tamura Ozaki ${ }^{1}$, Tatiana Emy Koike ${ }^{1}$, Thiago Alves Garcia ${ }^{1}$, Everton Alex Carvalho Zanuto ${ }^{1}$, José Carlos Silva Camargo Filho ${ }^{1}$, Regina Celi Trindade Camargo ${ }^{1}$, Patricia Monteiro Seraphim ${ }^{1}$

${ }^{1}$ Universidade Estadual Paulista, Faculdade de Ciências e Tecnologia, FCT/UNES, Presidente Prudente - SP. ${ }^{2}$ Universidade do Oeste Paulista, UNOESTE, Presidente Prudente - SP. ${ }^{3}$ Universidade Estadual de Campinas, Faculdade de Ciências Médicas, UNICAMP. E-mail: castoldi rc@yahoo.com.br

\section{RESUMO}

O presente estudo buscou analisar o efeito do exercício de força na composição corporal de ratos acometidos por obesidade, induzida por dieta hipercalórica. Foram utilizados 12 ratos, divididos em dois grupos: Controle Treinamento $(C T, n=6)$ e Obeso Treinamento (OT, $n=6)$. Os animais do grupo CT apresentaram valor reduzido do índice de Lee e IMC, além de menor quantidade de tecido adiposo epididimal em relação ao OT. Portanto conclui-se que esta forma de exercício pode auxiliar no combate a obesidade, entretanto, salienta-se que além do exercício, a dieta exerce papel fundamental na redução de peso corporal, sendo a associação de exercício e dieta adequada, a forma mais eficaz na redução do peso corporal.

Palavras-chave: exercício, composição corporal, tecido adiposo, obesidade, ratos.

\section{EFFECT OF STRENGTH EXERCISE IN BODY COMPOSITION OF OBESITY RATS}

\section{ABRSTRACT}

The present study has investigated the effect of strength excise on body composition of rats suffering from obesity induced by high calorie diet. Twelve rats were used, divided into two groups: Control Training (CT, $n=6$ ) and Obese Training (OT, $n=6)$. The animals of group CT shows lower value in BMI and Lee index, besides lower amount of epididymal adipose tissue than to OT. Thus it is concluded that this form of exercise can be useful against obesity, however, it is noted that besides the exercise, the diet plays a primordial role in reducing body weight and the association of exercise and proper diet is the most effective way in reducing body weight.

Keywords: exercise, body composition, adipose tissue, obesity, rats. 


\section{INTRODUÇÃO}

A obesidade é um problema para a saúde mundial, ocorrendo devido ao acumulo de gordura excessivo, podendo acarretar em graves doenças ${ }^{1}$. Ela está relacionada ainda ao surgimento de doenças crônico-degenerativas como diabetes, cardiopatias e hipertensão arterial, sendo a atividade física um fator positivo no combater a essa doença ${ }^{2}$.

Um fator desencadeante da obesidade é o estilo de vida sedentário, em que há alto consumo de calorias e baixo gasto energético, promovendo o acumulo de tecido adiposo ${ }^{3}$.

A redução de massa gorda está relacionada ao balanço energético negativo, em que o gasto energético é maior que o consumo, resultando na redução da massa gorda. Nesse contexto o exercício exerce um fator fundamental, propiciando maior gasto energético ${ }^{4}$.

Para induzir os animais à obesidade Panveloski-Costa et al., ${ }^{5}$ utilizaram com sucesso um protocolo de dieta hiperlipídica. Esse protocolo além de ser de fácil aplicação, ainda apresenta melhor correspondência com o tipo de obesidade que mais acomete o ser humano.

A dieta hiperlipídica promove aumento do tecido adiposo e do peso corporal de ratos ${ }^{6}$. Entretanto o exercício físico apresenta efeitos positivos em relação aos parâmetros de peso corporal, área de adipócitos e perfil lipídico em ratos obesos submetidos a esse tipo de dieta ${ }^{7}$.

Sendo assim, o presente estudo objetivou analisar os efeitos do treinamento de força na composição corporal de ratos obesos.

\section{MÉTODOS}

\section{Animais}

Foram utilizados 12 ratos Wistar machos, com 90 dias de idade, mantidos em gaiolas plásticas coletivas ( $30 \times 16 \times 19 \mathrm{~cm}$ ), com três a cinco animais por gaiola, sob temperatura média de $22 \pm 2{ }^{\circ} \mathrm{C}$ e ciclo claro/escuro de 12 horas, com o ciclo claro iniciando-se às 7:00hs. Os animais foram divididos aleatoriamente em dois grupos: Controle Treinamento (CT, $n=6)$, e Obeso Treinamento (OT, $n=6$ ). Todos os procedimentos experimentais utilizados foram aprovados pelo Comitê de Ética em Pesquisa da UNESP - Campus de Presidente Prudente, processo no 04/2011.

Os animais do grupo CT foram alimentados com ração padrão para roedores (marca Supra Lab ${ }^{\circledR}$ - Alisul Ind. Alimentos Ltda; São Leopoldo/RS, com composição de 25\% de proteínas, 3\% de lipídeos, 18\% e fibras, $11 \%$ de material mineral, $2 \%$ de cálcio e 0,5\% de fósforo) e água de torneira fornecida ad libitum. Já o grupo OT seguiu uma dieta hiperlipídica ${ }^{5}$ (dieta de cafeteria). 


\section{Treinamento Intermitente}

Foi utilizado o treinamento intermitente de força semelhante ao "Jump Squat", proposto por Tamaki, Uchiyama e Nakano ${ }^{8}$, com algumas adaptações. Utilizouse estimulação elétrica por meio de clipe metálico que envolveu a extremidade da cauda do animal, ligado a um eletroestimulador tipo Dualpex 961, da Quarker $^{\circledR}$, calibrado pelo Inmetro $^{\circledR}$.

Foram utilizados os seguintes parâmetros: frequência de $1 \mathrm{Hertz}(\mathrm{Hz})$, duração de 0,3 s com intervalo de 2 s entre cada estimulação elétrica, e a intensidade ajustada de forma que o animal executasse o movimento, variando de 3 a 6 miliamperes (mA). Esses parâmetros foram adotados por serem pulsos bidirecionais de média nula, não apresentando efeitos eletrolíticos, permitindo aplicações de longa duração sem risco de lesão aos tecidos. Com a estimulação os animais realizavam movimento de extensão completa da pata (joelho e tornozelo), levantando uma carga, posicionada no dorso do animal, por meio de um colete.

O protocolo de treinamento foi composto de três séries de 12 repetições, três vezes por semana, durante oito semanas. Foram realizadas três sessões de adaptação ao treino na primeira semana sem incremento de carga da seguinte forma: uma, duas e três séries de 12 repetições do primeiro ao terceiro dia respectivamente. Nas primeiras duas semanas, os animais realizaram o treino sem incremento de carga. A partir da terceira semana, uma carga equivalente a $50 \%$ do peso corporal (PC) foi imposta, até o final do protocolo de treinamento.

A carga era ajustada semanalmente de acordo com as variações do PC semanalmente. O movimento "jump squat" foi testado e validado como indutor de hipertrofia em animais semelhante à hipertrofia obtida em humanos levantadores de peso $^{9}$. O protocolo de treinamento ${ }^{5}$, iniciou-se juntamente com a dieta hiperlipídica, quando os animais atingiram a idade de adulto jovem; aos dois meses de idade.

\section{Procedimento Cirurgico}

Após 24 horas da última sessão de treinamento os animais foram medidos e pesados, sendo então eutanasiados, após anestesia (Xilasina e Quetamina 1:1; $60 \mathrm{mg} / \mathrm{kg}$ ). Em seguida foi feita uma incisão paramedial no abdômen para retirada do tecido adiposo epididimal.

\section{Índice de Lee}

Foi calculado em todos os animais, utilizando a relação entre raiz cúbica do peso corporal em gramas (g) pelo comprimento focinho-cóccix $(\mathrm{cm})$ e multiplicando-se por 
10, seguindo procedimento descrito por Novelli et al., ${ }^{10}$.

\section{Índice de Massa Corporal}

O Índice de Massa Corporal (IMC) foi calculado pela fórmula: Peso Corporal (g) / [Comprimento cabeça-nádega $(\mathrm{mm})]^{2}$. Tal procedimento foi reproduzido de acordo com os propostos por Novelli et al., ${ }^{9}$ e Sjögren et al., ${ }^{11}$.

\section{Tecido Adiposo Epididimal}

Após extração, o tecido adiposo epididimal (TecAdp) foi pesado em balança de precisão. Para a análise dos dados, foi determinado o seu percentual em relação ao peso do animal pela fórmula matemática: $\{[$ TecAdp (g) $x$ 100] / Peso (g)\}. Tal procedimento foi estabelecido como forma de correção da diferença da massa corporal entre os animais ${ }^{12}$.

\section{Análise Estatística}

Foi utilizado o Teste Shapiro-Wilk para a verificação da normalidade dos dados, e, o teste $t$-student para a comparação entre grupos. Todos os procedimentos adotaram o valor de significância $95 \% \quad(p<0,05)$. Os cálculos foram realizados com o aplicativo SPSS 17.0 for Windows ${ }^{\circledR}$.

\section{RESULTADOS}

Os resultados mostraram que o grupo OT apresentou uma maior quantidade de tecido adiposo quando comparado ao grupo CT (Tabela 1).

Da mesma forma o Índice de Lee, apresentou-se maior no grupo $\mathrm{OE}$ do que no grupo $C E$, o grupo OE também mostrou maior IMC que o grupo CE (Tabela 1).

Tabela 1. Valores de média \pm desvio padrão do tecido adipodo, índice de Lee e IMC.

\begin{tabular}{lcccc}
\hline Variáveis & CT & OT & $t$ & $p$-valor \\
\hline Tecido Adiposo & $2,636 \pm 1,054$ & $6,636 \pm 1,666$ & 4,968 & $0,001^{*}$ \\
Índice de Lee & $2,983 \pm 0,078$ & $3,106 \pm 0,168$ & - & 0,078 \\
IMC & $0,648 \pm 0,047$ & $0,756 \pm 0,112$ & - & $0,024^{*}$
\end{tabular}

* Diferença estatisticamente significante entre os grupos CE e OE.

\section{DISCUSSÃO}

Após a análise dos dados, foi observado que o grupo CT apresentou menores valores para as variáveis de Tecido
Adiposo, Lee e IMC, em comparação ao grupo OT.

Um estudo semelhante, realizado por Panveloski-Costa et al., ${ }^{5}$ evidenciaram o aumento tanto no peso quanto na massa 
adiposa dos animais que foram submetidos à dieta hipercalórica. Em contrapartida observou-se que o modelo de treinamento intermitente de força do tipo "Jump Squat" diminuiu os valores na composição corporal.

Nota-se que o exercício físico é uma das formas de se aumentar o gasto energético contribuindo assim para a diminuição da gordura corporal. Tamaki Uchiyama e Nakano ${ }^{8}$ observaram a perda de peso de gordura após a utilização do treinamento de força muscular. Assim, o método utilizado no presente estudo pode ser uma forma de se combater ou controlar a epidemiologia da obesidade.

Foi observado também que os animais exercitados apresentaram menores valores para o índice de Lee, um possível indicador de obesidade animal, que leva em consideração as variáveis de peso e comprimento, semelhante ao IMC utilizado para humanos. Nesse sentido, a diminuição desse índice sugere melhora no quadro da obesidade. A obesidade, em particular o excesso de adiposidade corporal, vem sendo fortemente associada à resistência insulínica, hipertensão e dislipidemia, colaborando para as elevadas taxas de mortalidade e morbidade em humanos ${ }^{13-14}$.

Outra variável que apresentou diminuição foi o IMC. Sabe-se que as medidas de peso e estatura corporal pode ser um indicador de obesidade e excesso de peso na população mundial ${ }^{3}$. Sendo assim salienta-se a importância de uma dieta adequada em conjunto com a prática de exercícios para uma mais eficaz diminuição da massa gorda e perda de peso, pois quando observamos que no presente estudo o exercício de forma isolada não obteve resultados tão significantes.

O presente estudo colabora com a literatura ao investigar o efeito do treinamento de força muscular na composição corporal de ratos Wistar. No entanto, algumas limitações devem ser levadas em consideração, como a mensuração da área de secção transversa das fibras musculares, avaliação pré treinamento e protocolo utilizado. Futuras investigações que possam abordar formas diferentes de treinamento podem vir a contribuir com a presente pesquisa.

\section{CONCLUSÃO}

Conclui-se que o grupo CT apresentou menores valores para as variáveis de Tecido Adiposo, Lee e IMC após a realização do treinamento de força muscular.

\section{REFERÊNCIAS}

1. Kopelman PG. Obesity as a medical problem . Nature 2000;404(6778):635-43.

2. Yancey AK, Fielding JE, Flores GR, Sallis JF, McCarthy WJ,Breslow L. Creating a Robust Public Health Infrastructure for Physical Activity Promotion. Am J Prev Med. 
2007;32(1):68-78.

http://dx.doi.org/10.1016/j.amepre.2006.08. $\underline{029}$

3. WORLD HEALTH ORGANIZATION. Obesity and overweight. WHO Global Strategy on Diet, Physical Activity and Health. Geneva: WHO, 2008.

4. Hill JO, Drougras H, Peters JC. Obesity treatment: can diet composition play a role? Ann Inter Med. 1993;119(7):694-697. http://dx.doi.org/10.7326/0003-4819-1197 Part 2-199310011-00013

5. Panveloski-Costa $A C$, Pinto Júnior $D A$, Brandão BB, Moreira RJ, Machado UF, Seraphim PM. Resistive training reduces inflammation in skeletal muscle and improves the peripheral insulin sensitivity in obese rats induced by hyperlipidic diet. Arq Bras Endocrinol Metab. 2011;55(2):155-163. DOI: $\quad$ http://dx.doi.org/10.1590/S000427302011000200008

6. Duarte ACGO, Fonseca DF, Manzoni MSJ, Soave CF, Fiorese MS, Damaso AR, et al., Dieta hiperlipídica e capacidade secretória de insulina em ratos. Rev Nutr. 2006;19(3):341348. DOI: http://dx.doi.org/10.1590/S1415$\underline{52732006000300005}$

7. Speretta GFF, Leite RD, Rosante MC, Andre RA, Duarte FO, Duarte, ACGO. The effects of exercise modalities on adiposity in obese rats. Clinics 2012;67(12):1469-1477. Doi: http://dx.doi.org/10.6061/clinics/2012(12)19

8. Tamaki T, Uchiyama S. Nakano S, et al. A weight-lifting exercise model for inducing hypertrophy in the hindlimb muscles of rats. Med Sci Sports Exerc. 1992;24:881-886. DOI: http://dx.doi.org/10.1249/00005768199208000-00009
9. Tamaki T, Akatsuka A, Tokunaga M, Ishige $\mathrm{K}$, Uchiyama S, Shiraishi T. Morphological and biochemical evidence of muscle hyperplasia following weight-lifting exercise in rats. Am J Physiol. 1997;273(1Pt1):C246-56.

10. Novelli ELB, Diniz YS, Galhardi CM, Ebaid GMX, Rodrigues HG, Mani $F$, et al. Anthropometrical parameters and markers of obesity in rats. Lab Anim. 2007;41:111-119. DOI:

http://dx.doi.org/10.1258/002367707779399 $\underline{518}$

11. Sjögren $K$, Hellberg $N$, Bohlooly-Y $M$, Savendahl $L$, Johansson MS, Berglindh $T$, et al. Body fat content can be predicted in vivo in mice using a modified dual-energy $x$-ray absorptiometry technique. J Nutr. 2001;131(11):2963-66.

12. Shi H, Strader AD, Woods SC, Seeley RJ. The effect of fat removal on glucose tolerance is depot specific in male and female mice. Am J Physiol Endocrinol Metab. 2007; 293(4):1012-20.

http://dx.doi.org/10.1152/ajpendo.00649.20 $\underline{06}$

13. Hotamisligil, Gökhan S. Inflammation and metabolic disorders. Nature 2006;444(7121):860-867. http://dx.doi.org/10.1038/nature05485

14. Shoelson, Steven E., Jongsoon Lee, and Allison B. Goldfine. Inflammation and insulin resistance. J Clin invest. 2006;116(7):17931801.

DOI: http://dx.doi.org/10.1172/JCI29069.

Recebido para publicação em 19/08/2014

Revisado em 26/08/2014

Aceito em 08/09/2014 